\title{
Survivin DEx3 as a biomarker of thyroid cancers: A study at the mRNA and protein level
}

\author{
JOANNA WALIGÓRSKA-STACHURA ${ }^{1 *}$, NADIA SAWICKA-GUTAJ ${ }^{1 *}$, MACIEJ ZABEL $^{2}$, \\ MIROSŁAW ANDRUSIEWICZ ${ }^{3}$, PAWEŁ GUT ${ }^{1}$, AGATA CZARNYWOJTEK ${ }^{1}$ and MAREK RUCHAŁA ${ }^{1}$ \\ ${ }^{1}$ Department of Endocrinology, Metabolism and Internal Medicine, Poznań University of Medical Sciences, \\ 60-355 Poznań; ${ }^{2}$ Department of Histology and Embryology, Poznań University of Medical Sciences, 60-781 Poznań; \\ ${ }^{3}$ Department of Cell Biology, Poznań University of Medical Sciences, 60-806 Poznań, Poland
}

Received July 13, 2016; Accepted November 23, 2016

DOI: $10.3892 / \mathrm{ol} .2017 .5713$

\begin{abstract}
Survivin and its splice variants DEx3 and 2B are involved in pathogenesis of numerous types of cancer. Proliferating cell nuclear antigen (PCNA) level correlates with cellular proliferation. The present study aimed to analyze the potential utility of survivin and its splice variants DEx 3 and $2 \mathrm{~B}$ as biomarkers for thyroid cancer. PCNA, survivin and its splice variants DEx3 and 2B expressions were analyzed in 22 tissue samples (15 thyroid cancers and 7 benign lesions) by reverse transcription-quantitative polymerase chain reaction and immunohistochemistry (IHC). There was significantly higher staining for survivin ( $\mathrm{P}=0.019)$, survivin $\mathrm{DEx} 3(\mathrm{P}=0.001)$, survivin $2 \mathrm{~B}(\mathrm{P}=0.0149)$ and PCNA $(\mathrm{P}=0.0237)$ in thyroid malignant tumors when compared with benign lesions. The receiver operating characteristics curve analysis has shown that the cut-off points of survivin IHC expression $>2$ [sensitivity $46.7 \%$; specificity $100 \%$; area under curve (AUC) $0.810 ; \mathrm{P}=0.0005$ ] and survivin DEx3 IHC expression $>0$ (sensitivity $86.7 \%$; specificity $100 \%$; AUC $0.933 ; \mathrm{P}<0.0001$ ) were the best predictors of thyroid malignancy. Additionally, PCNA staining $>1$ (sensitivity $93.3 \%$; specificity $71.4 \%$; AUC $0.790 ; \mathrm{P}=0.0243$ ) and survivin $2 \mathrm{~B}>2$ (sensitivity $46.7 \%$; specificity $100 \%$; AUC $0.824 ; \mathrm{P}=0.0002$ ) were the best predictors of thyroid cancer. In conclusion, the present study exhibited that survivin DEx3 expression has high specificity and sensitivity for discrimination between benign thyroid lesions and cancers. Survivin DEx3 may be considered
\end{abstract}

Correspondence to: Dr Nadia Sawicka-Gutaj, Department of Endocrinology, Metabolism and Internal Medicine, Poznan University of Medical Sciences, 49 Przybyszewski Street, 60-355 Poznań, Poland

E-mail: nyha@o2.pl

*Contributed equally

Key words: thyroid cancer, survivin, survivin DEx3, survivin 2B, expression, reverse transcription-quantitative polymerase chain reaction, immunohistochemistry a biological marker of thyroid malignancy and therefore applied in clinical practice.

\section{Introduction}

The incidence of thyroid cancer in developed countries has increased (1). Histopathological examination remains the gold standard for the diagnosis and classification of thyroid lesions excised during surgery (2). In addition, molecular markers of thyroid cancers may be used as diagnostic and even prognostic tools $(3,4)$. Certain markers may also be targets for non-conventional therapy.

Survivin, a protein belonging to the family of apoptosis inhibitors, is involved in cell-cycle regulation and proliferation $(5,6)$. Survivin overexpression has been observed in types of endocrine cancer including differentiated and medullary thyroid cancer, while was not upregulated in benign pituitary adenoma (7-16). However, survivin has not been proven to discriminate between thyroid follicular cancer and adenoma $(17,18)$. The two survivin splice variants $2 \mathrm{~B}$ and DEx3 demonstrate different biological properties. Survivin 2B is involved in apoptosis activation. By contrast, anti-apoptotic survivin DEx3 is suggested to be associated with tumor aggressiveness, advanced stage and poor prognosis in numerous types of cancer (5). We have previously reported survivin and its DEx3 mRNA variant overexpression in thyroid cancer $(19,20)$.

Another nuclear protein, proliferating cell nuclear antigen (PCNA), is also involved in replication, so its level correlates with cellular proliferation (21).

The aims of the present study were to confirm the findings on the overexpression of survivin and its DEx 3 variant in thyroid cancer at the protein level using immunohistochemical (IHC) staining, and to evaluate the role of survivin and its splice variants DEx 3 and 2B as potential biomarkers of thyroid malignancy. The present study additionally estimated the PCNA protein expression reflecting proliferative activity of tumors to better understand survivin and associated splice variant expression.

\section{Materials and methods}

Thyroid tissues. The present study group consisted of 15 thyroid cancer tissue samples: 5 from papillary thyroid 
cancers; 5 from medullary thyroid cancers; 4 from follicular thyroid cancers; and 1 undifferentiated thyroid cancer. In total, 7 thyroid tissue samples derived from benign lesions served as controls: 4 cases of colloid nodules and 3 cases of follicular adenomas. The thyroid tissues were obtained from patients who had undergone thyroidectomy due to several medical reasons. The resected thyroid tissue specimens were divided into 2 samples. The first was immediately submerged in an RNA protective medium and stored at $-80^{\circ} \mathrm{C}$ awaiting reverse transcription-quantitative polymerase chain reaction (RT-qPCR) analysis. The second sample was placed in a tissue processor, dehydrated and fixed in $10 \%$ neutral buffered formalin and embedded in paraffin for subsequent pathological examination and IHC staining. The present study was approved by the Ethics Committee of Poznań University of Medical Sciences (Poznań, Poland), and written informed consent was obtained from each patient.

$R N A$ isolation and RT. With the use of 3-Zone RNA Isolation Reagent (Novazym Polska s.c., Poznań, Poland) total cellular RNA was extracted from all tissue samples including controls, according to the manufacturer's protocol (the procedure was conducted twice for each sample). Prior to the second 3-Zone purifying step, the RNA was suspended in $40 \mu \mathrm{l}$ RNase-, DNase- and pyrogen-free $\mathrm{H}_{2} \mathrm{O}$, and $5 \mu \mathrm{l}$ DNase and DNA digestion buffer (Zymo Research Corp., Irvine, CA, USA) was added. The reaction mix was incubated at $25^{\circ} \mathrm{C}$ for $15 \mathrm{~min}$. Subsequent to the concentration and purity measuring processes (NanoDrop ND-1000 spectrophotometer; Thermo Fisher Scientific, Inc., Waltham, MA, USA), agarose gel electrophoresis was performed in order to verify RNA integrity. Isolated RNA was reverse transcribed or stored at $-80^{\circ} \mathrm{C}$ until additional analysis. The cDNA synthesis was carried out as previously described using $1 \mu \mathrm{g}$ of the RNA and universal oligo(d) $\mathrm{T}_{10}$ primer (Genomed, Warszawa, Poland) in the final volume reaction of $20 \mu \mathrm{l}$ (22). Complementary DNA was synthetized, according to the reverse transcriptase manufacturer's protocol, using $50 \mathrm{ng} / \mu 1$ total RNA, $5 \mathrm{pm} / \mu 1$ universal oligo(d) $\mathrm{T}_{10}$ primer, $10 \mathrm{U} / \mu 1$ Expand Reverse Transcriptase, 1X Expand Reverse Transcriptase Buffer, $10 \mathrm{U} / \mu 1$ RNasin (RNase inhibitor) and $1 \mathrm{pm} / \mu 1 \mathrm{dNTPs}$ (all Roche Molecular Diagnostics, Pleasanton, CA, USA). The mixture of RNA, oligo(d) $\mathrm{T}_{10}$ primer and water was incubated $10 \mathrm{~min}$ at $65^{\circ} \mathrm{C}$. Subsequently the samples were incubated on ice. The thermocycling conditions were as follows: $10 \mathrm{~min}$ at $25^{\circ} \mathrm{C} ; 30 \mathrm{~min}$ at $55^{\circ} \mathrm{C}$; and $5 \mathrm{~min}$ at $85^{\circ} \mathrm{C}$.The cDNA was immediately used for the qPCR analysis.

$q P C R$ analysis. To establish the expression levels of survivin (NCBI no., NM_001168), and its splice variants: BIRC5-DEx3 (NCBI no., NM_001012270.1) and BIRC5-2B (NCBI no., NM_001012271.1) TaqManÒ hydrolysis probe no. 36 (cat. no., 04687949001; Roche Applied Science, Manheim, Germany) and gene specific primers sets were used. The generated amplicon lengths and primers were: 88 bp for survivin sense, 5'-GCCCAGTGTTTCTTCTGCTT-3' and antisense, 5'-AACCGGACGAATGCTTTTTA-3'; BIRC5-2B sense, 5'-TCTGCTTCAAGGAGCTGGA-3' and antisense, 5'-AAA GTGCTGGTATTACAGGCGTA-3'; and 77 bp primers for BIRC5-DEx3 were sense, 3'-CAGTGTTTCTTCTGCTTC
AAGG-5' and antisense, 3'-CTTATTGTTGGTTTCCTT TGC-5'. To prevent product synthesis from genomic DNA, the primer sets were designed to genic region with intragenic fragments between 1,874 and 8,670 bp in length, depending on the survivin variant. The assays were designed in silico using ProbeFinder Software (version 2.50; Roche Applied Science) and applied in qPCR reactions. Human HPRT Gene Assay (cat. no., 05046157 001; Roche Applied Science) served as reference reaction. qPCR was performed as previously described in a total reaction volume of $20 \mu \mathrm{l}$ in a LightCycler ${ }^{\circledR} 2.0$ carousel based system (Roche Applied Science) (20). All reactions were made in triplicates with the hot start 1 X LightCycler ${ }^{\circledR}$ TaqMan $^{\circledR}$ Master mix (Roche Applied Science). The probes and primers concentration were set to $200 \mathrm{nM}$. The thermal profile was as follows: Pre-incubation step, $95^{\circ} \mathrm{C}, 10 \mathrm{~min} ; 45$ quantitation cycles (denaturation $95^{\circ} \mathrm{C}, 10 \mathrm{sec}$; annealing/elongation $60^{\circ} \mathrm{C}$, $30 \mathrm{sec}$; fluorescence data acquisition $72^{\circ} \mathrm{C}, 1 \mathrm{sec}$ ); and cooling step to $40^{\circ} \mathrm{C}$.

Reaction efficiency was calculated by comparing row data with the genes corresponding standard curve generated as described previously (22). Each reaction set included the negative no template control (total cellular RNA pool from different samples and isolation in RT reaction lack of transcriptase). As no contamination had been observed, the uracil-DNA glycosylase incubation step was omitted.

The qPCR raw data were collected and analyzed with the use of the LightCycler ${ }^{\circledR}$ Data Analysis (LCDA) Software (version 4.0.5.415; Roche Applied Science). Each sample was analyzed in a triplicate and the average threshold value $\mathrm{Cq}$ was calculated. The $2^{-\Delta \Delta C q}$ method was used (23). Comparing the data with standard curve and reference genes fluorescence data acquisition, the concentrations ratios $(\mathrm{Cr})$ were calculated for each patient and control sample.

IHC staining. Formalin fixed paraffin-embedded tissue specimens of thyroid cancers and benign lesions were cut in $5 \mu \mathrm{m}$ thick sections. IHC reactions, subsequent to non-specific antigen binding in blocking solution, were performed with the use of rabbit monoclonal anti-survivin antibody (dilution, 1:250; cat. no., ab76424; Abcam, Cambridge, UK), rabbit polyclonal anti-survivin DEx3 antibody (dilution, 1:250; cat. no., ab3731; Abcam), rabbit polyclonal anti-survivin 2B antibody (dilution, 1:250; cat. no., ab3729; Abcam) and rabbit monoclonal anti-PCNA antibody (dilution, 1:250; cat. no., ab92552; Abcam). Reactions were visualized through subsequent incubation with secondary biotinylated goat anti-rabbit immunoglobulin antibody (1:100; cat. no. E0432; Dako, Glostrup, Denmark), streptavidin-horseradish peroxidase complex (LSAB2-HRP anti-rabbit/mouse; cat. no. K0675; DAKO, Glostrup, Denmark) and 3,3'-diaminobenzidine chromogen. The assessment was performed using light microscopy via the Olympus CX41 microscope (Olympus Corporation, Tokyo, Japan). The intensity of survivin/survivin $2 \mathrm{~B} /$ survivin DEx3 expressions was evaluated using the modified semiquantitative immunoreactive score (IRS) approach, according to Remmele and Stegner (24). The percentage of positively stained cells (PP) is scored (range, $0-4$ points) and the staining intensity (SI) is scored (range, 0-3 points), and these scores are combined (IRS=SI x PP). The final score was presented on a five-point scale (range, 0-4 points): 1-2 IRS points=1 point; 3-4 
IRS points=2 points; $6-8$ IRS points=3 points; and 9-12 IRS points, 4 points.

Statistical analysis. Statistical analyses were performed using MedCalc for Windows, version 15.8 (MedCalc Software, Ostend, Belgium). $\mathrm{P}<0.05$ was considered to indicate a statistically significant difference. A comparison of the analyzed parameters between 2 groups was performed using the Mann-Whitney U test. The strength of the association between analyzed parameters was measured with Spearman's rank correlation coefficient test. Receiver operating characteristics (ROC) curves were calculated to determine the potential of analyzed parameters in order to discriminate between thyroid cancer and benign lesions. An optimal cut-off point was calculated according to the highest accuracy (minimal false negative and false positive results). The area under the ROC curve (AUC) was used to check the prognostic value of particular parameters.

\section{Results}

Patients and IHC staining. The median age of patients with thyroid cancer was 59 years [interquartile range (IQR) 41.5-67.5 years] and in patients with benign lesions was 52 years (IQR 35-54.5 years; $\mathrm{P}=0.0668$ ). Clinical data, including patient demographics, tumor histology and staging, are presented in Table I. A positive staining of survivin and survivin $2 \mathrm{~B}$ was found in $92.9 \%$ of the thyroid cancers samples and in $57.1 \%$ of controls. Survivin DEx3 was recognized in $85.7 \%$ of the thyroid cancer samples and in none of benign specimens. When PCNA expression was analyzed in the same group, positive staining was found in $100 \%$ of thyroid cancer specimens and in $57.1 \%$ of benign lesions. There was significantly higher staining for survivin $(\mathrm{P}=0.019)$, survivin $\mathrm{DEx} 3$ $(\mathrm{P}=0.001)$, survivin 2B $(\mathrm{P}=0.0149)$ and $\mathrm{PCNA}(\mathrm{P}=0.0237)$ in thyroid malignant tumors when compared with benign lesions.

Relationship between IHC and mRNA expressions. There was a strong correlation between survivin DEx3 mRNA and IHC expressions $(\mathrm{P}=0.0014 ; \mathrm{r}=0.639)$. There was also a trend for association between survivin mRNA overexpression and IHC upregulation $(\mathrm{P}=0.05 ; \mathrm{r}=0.421)$. The present study has not observed the correlation between survivin $2 \mathrm{~B}$ mRNA and protein expression $(\mathrm{P}=0.2149)$. A significant association between survivin DEx3 and IHC expressions of survivin $(\mathrm{P}=0.0007 ; \mathrm{r}=0.670)$, survivin $2 \mathrm{~B}(\mathrm{P}<0.0001 ; \mathrm{r}=0.763)$, and PCNA ( $\mathrm{P}=0.0024 ; \mathrm{r}=0.614)$ were also observed (Fig. 1). There were no correlations between tumor stage and survivin DEx3 mRNA and protein expressions.

IHC expression in the prognosis of thyroid malignancy. The ROC curve analysis has shown that the cut-off point of survivin IHC expression $>2$ (sensitivity $46.7 \%$; specificity $100 \%$; AUC $0.810 ; \mathrm{P}=0.0005$ ) and survivin DEx3 IHC expression $>0$ (sensitivity $86.7 \%$; specificity $100 \%$; AUC 0.933; $\mathrm{P}<0.0001$ ) were the best predictors of thyroid malignancy (Fig. 2). PCNA staining >1 (sensitivity 93.3\%; specificity 71.4\%; AUC 0.790; $\mathrm{P}=0.0243$ ) and survivin $2 \mathrm{~B}>2$ (sensitivity $46.7 \%$; specificity $100 \%$; AUC $0.824 ; \mathrm{P}=0.0002$ ) were the best predictors of thyroid cancers.
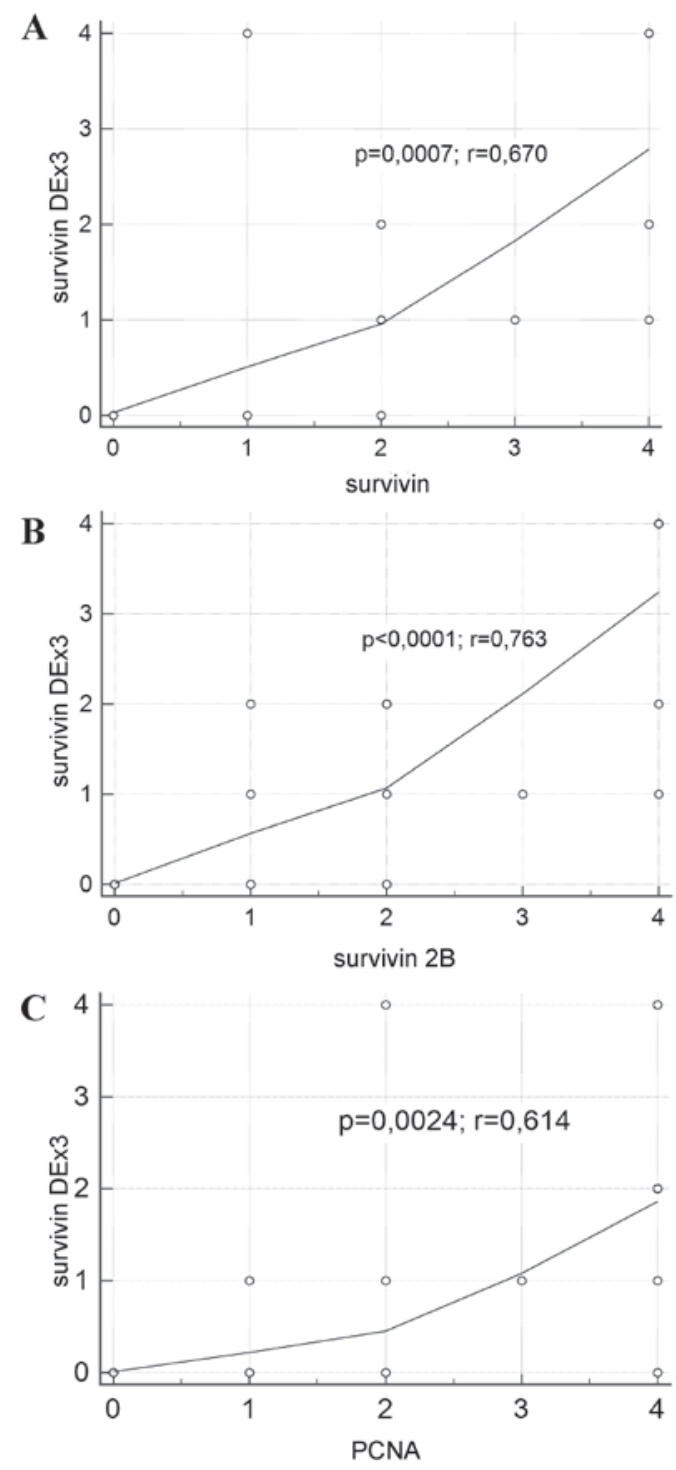

Figure 1. Positive association between survivin DEx3, and (A) survivin, (B) survivin 2B and (C) PCNA immunohistochemical expressions in benign and malignant thyroid lesions. The immunoreactive scores of the proteins were compared. PCNA, proliferating cell nuclear antigen.

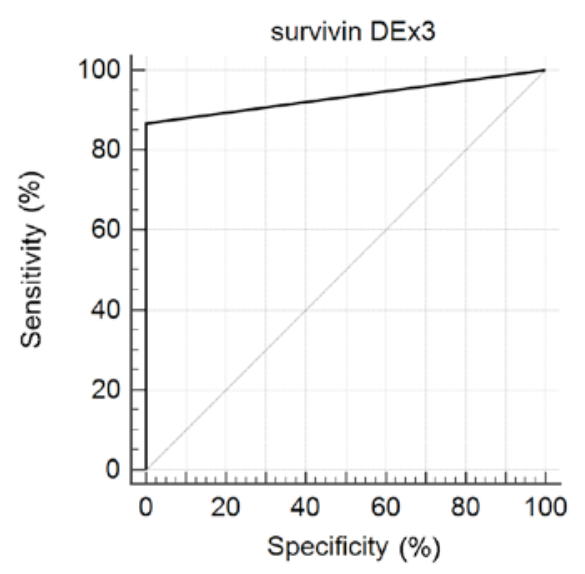

Figure 2. Survivin DEx3 immunohistochemical expression in the prognosis of thyroid malignancy. Survivin DEx3 IHC expression $>0$ was the best predictor of thyroid malignancy (sensitivity, $86.7 \%$; specificity, $100 \%$; area under the curve, 0.933; $\mathrm{P}<0.0001)$. 
Table I. Clinicopathological data of patients from the study and control groups.

\begin{tabular}{|c|c|c|c|c|c|}
\hline & Patients, $\mathrm{n}$ & Age, years & Gender & Histology, $\mathrm{n}$ & Staging, $n$ \\
\hline Thyroid cancer & 15 & Me 59 & F 13 & PTC 5 & T1- 4 \\
\hline & & (IQR 41.5-67.5) & M 2 & $\begin{array}{l}\text { FTC } 4 \\
\text { MTC } 5 \\
\text { UTC } 1\end{array}$ & $\begin{array}{l}\text { T2- } 3 \\
\text { T3- } 4 \\
\text { T4- } 4\end{array}$ \\
\hline Benign lesions & 7 & $\begin{array}{l}\text { Me } 52 \\
\text { (IQR 35-54.5) }\end{array}$ & $\begin{array}{l}\text { F } 4 \\
\text { M } 3\end{array}$ & $\begin{array}{l}\text { Colloid nodule } 4 \\
\text { Follicular adenoma } 3\end{array}$ & - \\
\hline
\end{tabular}

Me, median; T, tumor stage; IQR, interquartile range; F, female; M, male; PTC, papillary thyroid cancer; FTC, follicular thyroid cancer; MTC, medullary thyroid cancer; UTC, undifferentiated thyroid cancer.
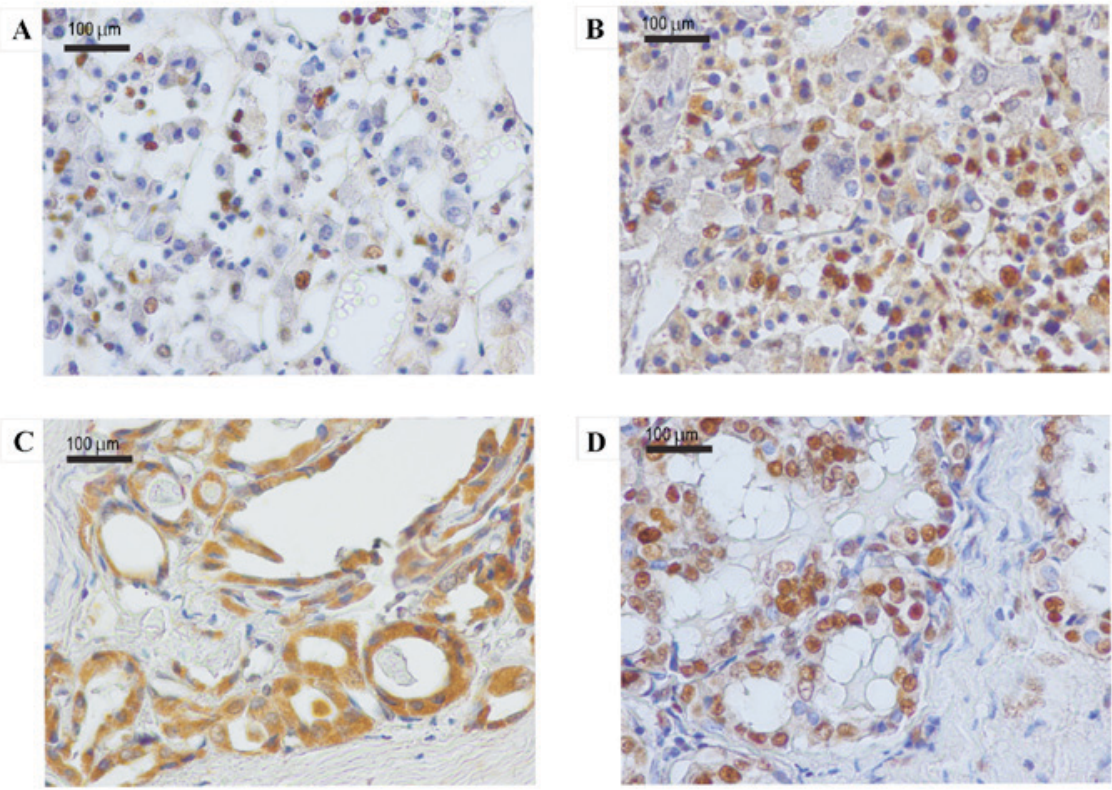

Figure 3. Immunohistochemical nuclear and cytoplasmic expression of (A) survivin, (B) survivin DEx3, (C) survivin 2B (D) and PCNA in cancer cells of follicular thyroid cancer. PCNA, proliferating cell nuclear antigen.

Fig. 3 shows cytoplasmic and nuclear staining of survivin, its splice variants DEx3 and 2B, and PCNA in thyroid cancer.

\section{Discussion}

In the present study, it was shown that survivin DEx3 expression has high specificity and sensitivity for discrimination between benign thyroid lesions and cancer. As a result, survivin DEx3 may be considered a biological marker of thyroid malignancy and therefore applied in clinical practice. To the best of our knowledge, this is the first study evaluating survivin DEx3 IHC expression in thyroid lesions. These findings are in agreement with our previous studies showing overexpression of survivin DEx3 $m R N A$ in thyroid cancer $(19,20)$. Using RT-qPCR, the present study also observed a positive association between the overexpression of survivin DEx3 mRNA and tumor aggressiveness. However, due to the limited sample size in the present study, the association between survivin DEx3 IHC overexpression and tumor stage and metastases is not confirmed.
Survivin protein overexpression in thyroid cancers has been previously reported (25). Pannone et al (26) observed enhanced cytoplasmic localization of survivin in differentiated thyroid cancer, whereas poorly differentiated and anaplastic thyroid cancer exhibited predominant nuclear protein staining by IHC. The authors hypothesized that this fact may be explained by the nuclear location of survivin splice variant DEx3. Recently, Selemetjev et al (27) reported stronger IHC staining of survivin in anaplastic in comparison with papillary thyroid cancer. Based on this observation, the authors suggested that survivin may perform a role in the progression of thyroid malignancy.

The present study also exhibited strong positive correlation between survivin DEx3 and PCNA expressions at the protein level in thyroid lesions. Upregulation of PCNA in thyroid cancer has been previously observed $(28,29)$. In comparison with PCNA staining the current study observed that survivin DEx3 expression exhibited higher specificity with similar sensitivity. Survivin DEx3 showed stronger staining compared with survivin and survivin $2 \mathrm{~B}$. These findings 
also support the potential clinical utility of survivin DEx3 upregulation in discrimination between malignant and benign types of thyroid tumor. This issue is particularly important among patients with thyroid nodules in whom the decision about thyroidectomy has to be made. Molecular biomarkers with high specificity and sensitivity could be helpful in the decision-making process, and certain patients may avoid unnecessary invasive procedures. Long-term observations may identify whether survivin DEx3 overexpression in thyroid cancer may be helpful in identifying patients with poor prognosis unresponsive to conventional therapy. Targeting of survivin gene may be a novel therapy in patients who did not respond to conventional treatment (30).

\section{Acknowledgements}

The present study was supported by The Polish Committee for Scientific Research Award (grant no., N N402 523640).

\section{References}

1. Davies L and Welch HG: Increasing incidence of thyroid cancer in the United States, 1973-2002. JAMA 295: 2164-2167, 2006.

2. JarząbB,DedecjusM,Handkiewicz-JunakD,LangeD,LewińskiA, Nasierowska-Guttmejer A, Ruchała M, Słowińska-Klencka D, Nauman J, Adamczewski Z, et al: Diagnostics and treatment of thyroid carcinoma. Endokrynol Pol 67: 74-107, 2016.

3. Kaczka K, Fendler W, Borowiec M, Młynarski W and Pomorski L: First one-step nucleic acid amplification testing in papillary thyroid cancer lymph nodes-a comparison with histopathology and real-time PCR. Endokrynol Pol 65: 422-430, 2014

4. Koziołek M, Bińczak-Kuleta A, Stepaniuk M, Parczewski M, Andrysiak-Mamos E, Sieradzka A, Safranow K, Osowicz-Korolonek L, Kiedrowicz B, Kram A, et al: Frequency assessment of BRAF mutation, KRas mutation, and RASSF1A methylation in nodular goitre based on fine-needle aspiration cytology specimens Ocena częstości występowania mutacji genów BRAF, KRas oraz. Endokrynol Pol 66: 384-393, 2015.

5. Waligórska-Stachura J, Jankowska A, Waśko R, Liebert W, Biczysko M, Czarnywojtek A, Baszko-Błaszyk D, Shimek V and Ruchała M: Survivin-prognostic tumor biomarker in human neoplasms-review. Ginekol Pol 83: 537-540, 2012.

6. Chen X, Duan N, Zhang C and Zhang W: Survivin and Tumorigenesis: Molecular mechanisms and therapeutic strategies. J Cancer 7: 314-323, 2016.

7. Waligórska-Stachura J, Andrusiewicz M, Sawicka-Gutaj N, Kubiczak M, Jankowska A, Liebert W, Czarnywojtek A, Waśko R, Blanco-Gangoo AR and Ruchała M: Evaluation of survivin splice variants in pituitary tumors. Pituitary 18: 410-416, 2015.

8. Werner TA, Tamkan-Ölcek Y, Dizdar L, Riemer JC, Wolf A Cupisti K, Verde PE, Knoefel WT and Krieg A: Survivin and XIAP: Two valuable biomarkers in medullary thyroid carcinoma. Br J Cancer 114: 427-434, 2016.

9. Vandghanooni S, Eskandani M, Montazeri V, Halimi M, Babaei E and Feizi MA: Survivin-deltaEx3: A novel biomarker for diagnosis of papillary thyroid carcinoma. J Cancer Res Ther 7: 325-330, 2011.

10. Cohen C, Lohmann CM, Cotsonis G, Lawson D and Santoianni R: Survivin expression in ovarian carcinoma: Correlation with apoptotic markers and prognosis. Mod Pathol 16: 574-583, 2003.

11. Monzó M, Rosell R, Felip E, Astudillo J, Sánchez JJ, Maestre J, Martín C, Font A, Barnadas A and Abad A: A novel anti-apoptosis gene: Re-expression of survivin messenger RNA as a prognosis marker in non-small-cell lung cancers. JClin Oncol 17: 2100-2104, 1999.

12. Grabowski P, Kühnel T, Mühr-Wilkenshoff F, Heine B, Stein H, Höpfner M, Germer CT and Scherübl H: Prognostic value of nuclear survivin expression in oesophageal squamous cell carcinoma. Br J Cancer 88: 115-119, 2003.
13. Sah NK and Seniya C: Survivin splice variants and their diagnostic significance. Tumour Biol 36: 6623-6631, 2015.

14. Liu S, Qi L, Yu Q, Song Y, Han W, Zu X, Jiang S, Yuan J, Zeng F and Xie Y: Survivin and HLA-I expression predicts survival of patients with clear cell renal cell carcinoma. Tumour Biol 35: 8281-8288, 2014

15. Lv S, Turlova E, Zhao S, Kang H, Han M and Sun HS: Prognostic and clinicopathological significance of survivin expression in bladder cancer patients: A meta-analysis. Tumour Biol 35: 1565-1574, 2014

16. Yazdani N, Sayahpour FA, Haghpanah V, Amiri P, Shahrabi-Farahani M, Moradi M, Mirmiran A, Khorsandi MT, Larijani B, Mostaan LV and Amoli MM: Survivin gene polymorphism association with papillary thyroid carcinoma. Pathol Res Pract 208: 100-103, 2012.

17. Jang MH, Jung KC and Min HS: The diagnostic usefulness of HMGA2, Survivin, CEACAM6, and SFN/14-3-3 $\delta$ in follicular thyroid carcinoma. J Pathol Transl Med 49: 112-117, 2015.

18. Ito Y, Yoshida H, Uruno T, Nakano K, Miya A, Kobayashi K, Yokozawa T, Matsuzuka F, Matsuura N, Kakudo K, et al: Survivin expression is significantly linked to the dedifferentiation of thyroid carcinoma. Oncol Rep 10: 1337-1340, 2003.

19. Waligórska-Stachura J, Andrusiewicz M, Sawicka-Gutaj N, Biczysko M, Jankowska A, Kubiczak M, Czarnywojtek A, Wrotkowska E and Ruchała M: Survivin delta Ex3 overexpression in thyroid malignancies. PLoS One 9: e100534, 2014.

20. Sawicka-Gutaj N, Waligórska-Stachura J, Andrusiewicz M, Biczysko M, Sowiński J, Skrobisz J and Ruchała M: Nicotinamide phosphorybosiltransferase overexpression in thyroid malignancies and its correlation with tumor stage and with survivin/ survivin DEx3 expression. Tumor Biol 36: 7859-7863, 2015

21. Wang SC: PCNA: A silent housekeeper or a potential therapeutic target? Trends Pharmacol Sci 35: 178-186, 2014.

22. Andrusiewicz M, Słowikowski B, Skibińska I, Wołuń-Cholewa M and Dera-Szymanowska A: Selection of reliable reference genes in eutopic and ectopic endometrium for quantitative expression studies. Biomed Pharmacother 78: 66-73, 2016.

23. Livak KJ and Schmittgen TD: Analysis of relative gene expression data using real-time quantitative PCR and the 2(-Delta Delta C(T)) method. Methods 25: 402-408, 2001.

24. Remmele W and Stegner HE: Recommendation for uniform definition of an immunoreactive score (IRS) for immunohistochemical estrogen receptor detection (ER-ICA) in breast cancer tissue. Pathologe 8: 138-140, 1987.

25. Zhang HY, Meng X, Du ZX, Fang CQ, Liu GL, Wang HQ and Deng WW: Significance of survivin, caspase-3, and VEGF expression in thyroid carcinoma. Clin Exp Med 9: 207-213, 2009.

26. Pannone G, Santoro A, Pasquali D, Zamparese R, Mattoni M, Russo G, Landriscina M, Piscazzi A, Toti P, Cignarelli M, et al: The role of survivin in thyroid tumors: Differences of expression in well-differentiated, non-well-differentiated, and anaplastic thyroid cancers. Thyroid 24: 511-519, 2014.

27. Selemetjev SA, Savin SB, Paunovic IR, Tatic SB and Cvejic D: Changes in the expression pattern of apoptotic molecules (galectin-3, Bcl-2, Bax, survivin) during progression of thyroid malignancy and their clinical significance. Wien Klin Wochenschr 127: 337-344, 2015.

28. Cvejic D, Savin S, Petrovic I, Selemetjev S, Paunovic I, Tatic S and Havelka M: Galectin-3 and proliferating cell nuclear antigen (PCNA) expression in papillary thyroid carcinoma. Exp Oncol 27: 210-214, 2005.

29. Cvejic D, Selemetjev S, Savin S, Paunovic I, Petrovic I and Tatic S: Apoptosis and proliferation related molecules (Bcl-2, Bax, p53, PCNA) in papillary microcarcinoma versus papillary carcinoma of the thyroid. Pathology 40: 475-480, 2008.

30. Chen Z, Liu N, Zhu G, Dralle H and Hoang-Vu C: Targeting of the anti-apoptotic gene survivin in human thyroid carcinoma. Int J Mol Med 30: 465-472, 2012. 